\title{
A HYBRID ASYMPTOTIC-FINITE ELEMENT METHOD FOR STIFF TWO-POINT BOUNDARY VALUE PROBLEMS*
}

\author{
R. C. Y. CHIN $\dagger$ AND R. KRASNY $\dagger$
}

\begin{abstract}
An accurate and efficient numerical method has been developed for a nonlinear stiff second order two-point boundary value problem. The scheme combines asymptotic methods with the usual solution techniques for two-point boundary value problems. A new modification of Newton's method or quasilinearization is used to reduce the nonlinear problem to a sequence of linear problems. The resultant linear problem is solved by patching local solutions at the knots or equivalently by projecting onto an affine subset constructed from asymptotic expansions. In this way, boundary layers are naturally incorporated into the approximation. An adaptive mesh is employed to achieve an error of $O\left(1 / N^{2}\right)+O(\sqrt{\varepsilon})$. Here, $N$ is the number of intervals and $\varepsilon \ll 1$ is the singular perturbation parameter. Numerical computations are presented.
\end{abstract}

Key words. projection method, quasilinearization, asymptotic expansions, boundary layers, singular perturbations, two-point boundary value problems, $\gamma$-elliptic splines

1. Introduction. We treat the following stiff boundary value problem:

$$
\begin{aligned}
& \varepsilon y^{\prime \prime}=f(x, y), \quad a<x<b, \quad f(x, y) \in C^{2}[(a, b) \times R], \\
& f_{y}(x, y)>0, \quad 0<\varepsilon \ll 1,
\end{aligned}
$$

with boundary conditions:

$$
\begin{aligned}
& a_{0} y(a)-a_{1} y^{\prime}(a)=\alpha, \\
& b_{0} y(b)+b_{1} y^{\prime}(b)=\beta .
\end{aligned}
$$

Under the assumptions that $a_{0} a_{1}>0, b_{0} b_{1}>0$ and $\left|a_{0}\right|+\left|b_{0}\right|>0$ Keller [26] and Bernfield and Lakshmikantham [8] have shown that the boundary value problem (BVP) has a unique solution. Using singular perturbation theory, O'Malley [31], [32] has proved that boundary layers occur at either or both boundaries depending on whether the solution of the reduced problem:

$$
f(x, z(x))=0
$$

satisfies the boundary conditions (1.2) and (1.3). In particular, Fife [18] and Bris [12] show that for a Dirichlet problem there are boundary layers at both boundaries.

This class of stiff or singular perturbation boundary value problems appears in many physical applications, for example, the confinement of a plasma column by radiation pressure (Troesch [42]), the theory of gas porous electrodes (Gidaspow et al. [22], Markin et al. [28]), the performance of catalytic pellets (Aris [3]) and in geophysical fluid dynamics (Carrier [14]).

Our method combines quasilinearization (Newton's method) in function space, asymptotic expansions and patching of local solutions. Quasilinearization is applied to reduce the nonlinear problem to a sequence of linear problems (Ascher, Christiansen and Russell [4], deBoor and Swartz [10], Russell and Shampine [40]). This is achieved in our scheme by interpolating $f(x, y)$ by a piecewise linear function of $y$ for fixed $x$. This step differs from the usual quasilinearization in that the resulting BVP has

* Received by the editors January 4, 1981, and in revised form June 7, 1982. This work was performed under the auspices of the U.S. Department of Energy by the Lawrence Livermore National Laboratory under contract No. W-7405-ENG-48.

† University of California, Lawrence Livermore National Laboratory, Livermore, California 94550.

‡ Graduate student, Department of Mathematics, University of California, Berkeley, California 94720. 
discontinuous coefficients. We use asymptotic methods to construct the local solutions in each subinterval. This should be compared with other asymptotic methods which also seek to capture the essential behavior of the differential equation (Flaherty and Mathon [19], Flaherty and O'Malley [20], deGroen and Hemker [23], deGroen [24], Hemker [25], Yarmish [45]). The local solutions are patched together by requiring that the computed solution and its derivative be continuous at the knots (Rose [38], Shampine [42]). This assures the computed solution is globally twice differentiable in an asymptotic sense.

This patching process for solving the linearized BVP is equivalent to a modified Galerkin method. This involves projection onto an affine subset constructed from the $\gamma$-elliptic splines of Schultz [41]. The computed solution, however, is based on $\gamma_{\varepsilon}$-splines which are asymptotic approximations of these. The $\gamma_{\varepsilon}$-splines become more accurate as $\varepsilon \rightarrow 0$ with no corresponding increase in calculational expense. This property is inherent in methods based on asymptotic expansions and is absent in finite difference and finite element methods based on polynomials (Abrahamsson [1], Berger et al., [6], Kellogg and Tsan [27], Osher [34]).

Another novel feature of our method is the mesh selection strategy. The interpolation error is proportional to $f_{y y}(\Delta y)^{2}$ which leads to a criterion for how the $y$-mesh should be chosen. This contrasts with the usual methods which specify the $x$-mesh. We consider both a uniform $y$-mesh and a $y$-mesh resulting from equally distributing the interpolation error (deBoor [9]). In either case, an iteration is introduced to construct the numerical solution and the grid structure. The resulting error depends only on the number of intervals $O\left(1 / N^{2}\right)$ rather than their size. This facet of the method appears to be new.

Our study has been influenced by Pruess' method of solving linear boundary value problems by approximating the coefficients [36]. The essence of Pruess' method lies in the ability of the approximation to capture the essential behavior of the solution. This is because the coefficients of the DE can be well approximated locally by polynomials while the solution cannot. In this light, our method may be viewed as solving nonlinear boundary value problems by approximating terms of the differential equation.

A detailed numerical analysis of the error and convergence properties will be presented in a forthcoming paper. However, some of the preliminary error analysis is presented to motivate the adaptive mesh selection. In $\S 2$, we describe the numerical algorithm. In $\S 3$, we discuss the error introduced by quasilinearization. In $\S 4$, we present the adaptive mesh technique and the solution of the algebraic system. In $\S 5$, we describe the construction of the $\gamma$-elliptic splines using asymptotics. In $\S 6$, computations are presented. Finally, conclusions and generalizations are presented in $\S 7$.

2. Derivation of the numerical method. We begin by replacing $f(x, y)$ by $I_{f}(x, y)$, its piecewise linear interpolant with respect to a $y$-mesh $\left(y_{i}, i=0,1, \cdots, N\right)$ for fixed $x$ :

$$
\begin{aligned}
I_{f}(x, y) & =f\left(x, y_{i-1}\right)+\left(y-y_{i-1}\right)\left[y_{i-1}, y_{i}\right] f, \quad y_{i-1}<y<y_{i}, \\
& =g_{0_{i}}\left(x ; y_{i-1}, y_{i}\right)+g_{1_{i}}\left(x ; y_{i-1}, y_{i}\right) y, \quad i=1, \cdots, N,
\end{aligned}
$$

where

$$
\left[y_{i-1}, y_{i}\right] f=\left(f\left(x, y_{i}\right)-f\left(x, y_{i-1}\right)\right) /\left(y_{i}-y_{i-1}\right) .
$$

We will solve the linear BVP $\varepsilon y^{\prime \prime}=I_{f}(x, y)$ together with boundary conditions (1.2)-(1.3) by a "patching" or multiple shooting process. With respect to an $x$-mesh, 
$a=x_{0}<x_{1}<\cdots<x_{N}=b$, define the following elliptic differential operators, $E_{i} y=$ $-\varepsilon y^{\prime \prime}+g_{1_{i}} y$, for $x_{i-1}<x<x_{i}$. On each interval we consider the linear BVP:

$$
E_{i} y=-g_{0_{i}}
$$

with boundary conditions given by

$$
\begin{aligned}
& a_{0} y(a)-a_{1} y^{\prime}(a)=\alpha, \quad y\left(x_{1}\right)=y_{1}, \quad i=1, \\
& y\left(x_{i-1}\right)=y_{i-1}, \quad y\left(x_{i}\right)=y_{i} \quad \text { for } i=2, \cdots, N-1, \\
& b_{0} y(b)+b_{1} y^{\prime}(b)=\beta, \quad y\left(x_{N-1}\right)=y_{N-1} \quad \text { for } i=N .
\end{aligned}
$$

It will sometimes be convenient to write these equations collectively as

$$
E y=-\varepsilon y^{\prime \prime}+g_{1} y=-g_{0} \text {. }
$$

Let $u_{i}(x), v_{i}(x)$ be the basis for the null space for $E_{i}$ normalized so that

$$
u_{i}\left(x_{i-1}\right)=v_{i}\left(x_{i}\right)=0 \text { and } u_{i}\left(x_{i}\right)=v_{i}\left(x_{i-1}\right)=1 .
$$

Let $y_{p_{i}}$ be the particular solution of the nonhomogeneous BVP

$$
\begin{aligned}
& E_{i} y_{p_{i}}=-g_{0_{i}}, \\
& y_{p_{i}}\left(x_{i-1}\right)=y_{p_{i}}\left(x_{i}\right)=0, \quad x_{i-1}<x<x_{i} .
\end{aligned}
$$

Then the solution of $(2.1)$ is given by:

$$
y\left(x ; y_{i-1}, y_{i}\right)=y_{i} u_{i}(x)+y_{i-1} v_{i}(x)+y_{p_{i}}(x), \quad x_{i-1}<x<x_{i} .
$$

There are $2 N$ degrees of freedom from $\left\{y_{i}\right\} i=0,1, \cdots, N$ and $\{x\}_{k} k=$ $1,2, \cdots, N-1$. By requiring that the solution (2.5) satisfies the boundary conditions (2.2) and is continuously differentiable at the knots $x_{i}, i=1, \cdots, N-1$, we obtain a nonlinear algebraic system relating the $x_{i}$ and $y_{i}$. With the remaining $N-1$ degrees of freedom we may prescribe $N-1$ additional relations to uniquely determine the $2 N$ quantities. This prescription should achieve the goal of improving efficiency and accuracy and will be discussed later.

The condition that $y \in C^{1}[a, b]$ is equivalent to equality of the derivatives from adjacent intervals at the knots:

$$
y^{\prime}\left(x_{i}^{-} ; y_{i-1}, y_{i}\right)=y^{\prime}\left(x_{i}^{+} ; y_{i}, y_{i+1}\right) \text {. }
$$

Differentiating (2.5) we obtain:

$$
\begin{aligned}
& v_{i}^{\prime}\left(x_{i}\right) y_{i-1}+\left[u_{i}^{\prime}\left(x_{i}\right)-v_{i+1}^{\prime}\left(x_{i}\right)\right] y_{i}-u_{i+1}^{\prime}\left(x_{i}\right) y_{i+1} \\
& =y_{p_{i+1}}^{\prime}\left(x_{i}\right)-y_{p_{i}}^{\prime}\left(x_{i}\right), \quad i=1,2, \cdots, N-1 .
\end{aligned}
$$

At the boundary nodes $x_{0}=a$ and $x_{N}=b$ we require that the boundary conditions (1.2)-(1.3) be satisfied. This gives:

$$
\begin{aligned}
& {\left[a_{0}-a_{1} v_{1}^{\prime}(a)\right] y_{0}-a_{1} u_{1}^{\prime}(a) y_{1}=\alpha+a_{1} y_{p_{1}}^{\prime}(a),} \\
& v_{N-1}^{\prime}(b) y_{N-1}+\left[b_{0}+b_{1} u_{N}^{\prime}(b)\right]=\beta-b_{1} y_{p_{N}}^{\prime}(b) .
\end{aligned}
$$

Equations (2.6)-(2.7) can be written in the form:

$$
A(x, y) y=h(x, y),
$$

where $y=\left(y_{0}, y_{1}, \cdots, y_{N}\right)^{T}, x=\left(x_{0}, x_{1}, \cdots, x_{N}\right)^{T}$.

Here $h$ is an $N+1$ component vector and $A$ is a symmetric diagonally dominant tridiagonal matrix of dimension $N+1$. $A$ has positive diagonal and negative offdiagonal elements and accordingly is a Stieltjes matrix. These properties follow from 
$f_{y}>0$ and from the construction of the basis functions $u_{i}$ and $v_{i}$. Symmetry of $A$ may be demonstrated by noting that the Wronskian of $u, v$ is constant in each interval $x_{i-1}<x<x_{i}$. Moreover, $y \in C^{2}[a, b]$ since $I_{f}(x, y) \in C[a, b]$. It should also be noted that the linearized problem $(2.2)-(2.3)$ is solved exactly by the above patching procedure.

The particular solution $y_{p_{i}}$ may be expressed by the usual variation of parameters formula since the Green's function for the Dirichlet problem for (2.1) exists. Thus we have:

$$
y_{p_{i}}=\frac{v_{i}}{c} \int_{x_{i-1}}^{x} u_{i} g_{0_{i}} d \xi+\frac{u_{i}}{c} \int_{x}^{x_{i}} v_{i} g_{0_{i}} d \xi
$$

where $c=\varepsilon$ Wronskian $\left(u_{i}, v_{i}\right)$

We now describe how (2.8) can be obtained by a Galerkin procedure. Let $S\left(E,\left\{x_{i}\right\}\right)$ be the space of $\gamma$-elliptic splines generated by the basis $w_{i}$ :

$$
\begin{aligned}
& w_{0}(x)= \begin{cases}v_{1}(x), & x \in\left(x_{0}, x_{1}\right), \\
0 & \text { otherwise, }\end{cases} \\
& w_{i}(x)= \begin{cases}u_{i}(x), & x \in\left(x_{i-1}, x_{i}\right), \\
v_{i}(x), & x \in\left(x_{i}, x_{i+1}\right), \quad i=1,2, \cdots, N-1, \\
0 & \text { otherwise, }\end{cases} \\
& w_{N}(x)= \begin{cases}u_{N}(x), & x \in\left(x_{N-1}, x_{N}\right), \\
0 & \text { otherwise. }\end{cases}
\end{aligned}
$$

Schultz [41] considered the problem of interpolating in spaces generated by such splines called $\gamma$-elliptic splines, where $\gamma$ is the smallest eigenvalue of the operator $E$. Note that our splines depend on both the $x$ and $y$ meshes.

Consider the problem of finding an element $y$ contained in the affine subset $\left[y_{p}\right] \oplus S\left(E,\left\{x_{i}\right\}\right)$, which satisfies $(2.1)$ along with the boundary conditions (2.2). Let

$$
a(u, v)=\frac{1}{\varepsilon} \int_{a}^{b}\left(\varepsilon u^{\prime} v^{\prime}+g_{1} u v\right) d x, \quad(u, v)=\frac{1}{\varepsilon} \int_{a}^{b} u v d x .
$$

A discretized weak form of this problem is: Find $y \in\left[y_{p}\right] \oplus S\left(E,\left\{x_{i}\right\}\right)$ such that

$$
a(y, w)=\left(g_{0}, w\right)+\left(-a_{0} y(a)+\alpha\right) w(a) / a_{1}-\left(b_{0} y(b)+\beta\right) w(b) / b_{1}
$$

for all $w \in S\left(E,\left\{x_{i}\right\}\right)$.

In case $a_{1}$ or $b_{1}=0$, we have Dirichlet boundary conditions and another appropriate weak form (Aubin [5]) holds. In any case equations (2.10) form a nonlinear system which is identical to (2.6)-(2.7). In verifying this, the following formulas are useful:

$$
\begin{aligned}
& a\left(y, w_{i}\right)=y_{i+1} w_{i}^{\prime}\left(x_{i+1}^{-}\right)+y_{i}\left(w_{i}^{\prime}\left(x_{i}^{-}\right)-w_{i}^{\prime}\left(x_{i}^{+}\right)\right)-y_{i-1} w_{i}^{\prime}\left(x_{i-1}^{+}\right), \\
& \left(g_{0}, w_{i}\right)=y_{p_{i+1}}^{\prime}\left(x_{i}^{+}\right)-y_{p_{i}}^{\prime}\left(x_{i}^{-}\right) \text {for } i=1, \cdots, N-1
\end{aligned}
$$

with similar formulas for $w_{0}, w_{N}$.

Thus the matrix elements in (2.8) are recognized as simply $a\left(w_{i}, w_{j}\right)$, and it follows that the matrix is symmetric, positive definite and tridiagonal.

The usual Galerkin method projects $y$ onto a subspace of $C^{0}[a, b]$. We call our procedure a modified Galerkin method because it projects $y$ onto an affine subset, i.e., a translated linear subspace. In other words, we can view our modified Galerkin 
method as applying the usual Galerkin method to $y-y_{p}$. It is striking that since $a\left(y_{p}, w_{i}\right)=0$, both Galerkin and modified Galerkin give the same set of discretized equations. The difference is that projecting onto the affine subset solves the linearized problem (2.3) exactly.

The equivalence of the "patching" procedure and the modified Galerkin method is another example of the interdependence of projection methods for two-point BVP as discussed by Reddien [36].

3. Discussion of the error due to quasilinearization. In this section we briefly discuss the error in order to motivate the mesh selection procedure. A detailed numerical analysis of the method will be presented in a forthcoming paper. For present purposes, a few of the preliminary results will be discussed.

For definiteness, let $y$ denote the exact solution and let it satisfy $\varepsilon y^{\prime \prime}=f(x, y)$ together with boundary conditions (1.2)-(1.3). Our method makes two main approximations to the original nonlinear BVP in order to arrive at the computed solution. We list these approximations along with their defining properties:

1 . Let $z$ be the $\gamma$-elliptic spline solution and satisfy

$$
\varepsilon z^{\prime \prime}=I_{f}\left(x, z ; z_{i}\right) \text { together with boundary conditions, }
$$

where $I_{f}\left(x, z ; z_{i}\right)=g_{0}\left(x ; z_{i}\right)+g_{1}\left(x, z_{i}\right) z$ is the linear interpolation of $f$ with knots $z_{i}$.

2. Let $z_{\varepsilon}$ be the actual computed solution, which is an asymptotic approximation to $z$ as will be explained in $\S 5$.

As seen in $\S 2$, an equivalent formulation of $(3.1)$ is

$$
\begin{aligned}
& A(x, z) z=h(x, z), \quad x=\left\{x_{i}\right\}^{T}, \quad z=\left\{z_{i}\right\}^{T}, \\
& z(x)=z_{i-1} w_{i-1}(x)+z_{i} w_{i}(x)+z_{p_{i}}(x) \quad \text { on }\left[x_{i-1}, x_{i}\right],
\end{aligned}
$$

where $w_{i}$ is the normalized $\gamma$-elliptic spline basis function at $x_{i}$ and $z_{p_{i}}$ is defined as in (2.4).

We will show that $|y-z|<c / N^{2}$. This is the error from the linear interpolation. It follows from asymptotic error bounds (Olver [29], [30]) that $z=z_{\varepsilon}(1+\zeta)$, where $|\zeta|=O(\sqrt{\varepsilon})$ as $\varepsilon \rightarrow 0$.

Using these two bounds we conclude that the pointwise error in the method can be bounded by a sum of two terms:

$$
\left|y-z_{\varepsilon}\right|<|y-z|+\left|z_{\varepsilon}\right||\zeta|
$$

thus we have $\sup \left|y-z_{\varepsilon}\right|<O\left(1 / N^{2}\right)+O(\sqrt{\varepsilon})$. Two important special cases deserve to be singled out:

(1) For an autonomous equation $\varepsilon y^{\prime \prime}=f(y)$, our method solves the linearized problem exactly. Thus, $z=z_{\varepsilon}$, and the second term in the error bound is not present. We expect our method to be competitive in this case even for $\varepsilon$ not small.

(2) For a linear BVP the first term in the error bound is not present. In this case our method simply reduces to the Liouville-Green or WKB asymptotic solution (Olver [29]).

We will discuss the first term now and reserve a discussion of the second term until the asymptotics have been introduced.

Define $\phi=y-z$. Subtracting the two equations for $y$ and $z$ we get

$$
\varepsilon \phi^{\prime \prime}=f(x, y)-I_{f}\left(x, z ; z_{i}\right) \text {. }
$$


The right-hand side can be written

$$
\begin{aligned}
f(x, y)-I_{f}\left(x, z ; z_{i}\right) & =f(x, y)-f(x, z)+f(x, z)-I_{f}\left(x, z ; z_{i}\right) \\
& =f_{y}\left(x, y^{*}\right)(y-z)+f(x, z)-I_{f}\left(x, z ; z_{i}\right)
\end{aligned}
$$

for some $y^{*}$ between $y$ and $z$. Thus $\phi$ satisfies the singular perturbation problem with homogeneous boundary conditions,

$$
\varepsilon \phi^{\prime \prime}-f_{\mathrm{y}}\left(x, y^{*}\right) \phi=f(x, z)-I_{f}\left(x, z ; z_{i}\right) .
$$

The driving term on the right-hand side is simply the error due to the linear interpolation and can be bounded by a standard result of approximation theory. For a Dirichlet problem, we can use the maximum principle (Dorr, Parter and Shampine [16], Protter and Weinberger [35]) to obtain the following a posteriori error bound.

LEMMA 1. Let

$$
\begin{aligned}
D & =[a, b] \times \text { range of exact solution, } \quad m^{2}=\min f_{\mathrm{y}} \quad \text { on } D, \\
M & =\max \left|f_{y y}(x, z(x))\right| \quad \text { on }[a, b], \quad \Delta z=\max \left|z_{i}-z_{i-1}\right|, \\
\kappa(x) & =1-\left\{\sinh \left[\frac{m(x-a)}{\sqrt{\varepsilon}}\right]+\sinh \left[\frac{m(b-x)}{\sqrt{\varepsilon}}\right]\right\} / \sinh \left[\frac{m(b-a)}{\sqrt{\varepsilon}}\right] .
\end{aligned}
$$

Then

$$
|\phi(x)|<(M / 8 m) \Delta z^{2} \kappa(x) .
$$

LEMMA 2.

$$
\begin{aligned}
& \text { If } f_{y y}(x, z(x))>0 \text { on }[a, b] \text { then } \phi>0 . \\
& \text { If } f_{y y}(x, z(x))<0 \text { on }[a, b] \text { then } \phi<0 .
\end{aligned}
$$

The comparison function $\kappa(x)$ is the solution of $\varepsilon \kappa^{\prime \prime}-m^{2} \kappa=-m^{2}$ with homogeneous Dirichlet boundary condition. It is strictly less than 1 and has boundary layers at $x=a, b$. Thus we expect a smaller error closer to the boundary. With further assumptions on the boundary condition parameters a similar bound holds for the Robin problem. Here, the function $\kappa(x)$ must be modified to account for Robin boundary conditions (Friedman [21], Stakgold [43]).

The form of the error bound suggests the following mesh selection strategy. For a given number of mesh intervals $N$, uniformly spaced $z_{i}$ points will produce an error bound of the form $c / N^{2}$. Another possibility is to choose the $z_{i}$ points to equally distribute the interpolation error in the sense of deBoor [9], which also gives a $c / N^{2}$ bound. In either case the constant $c$ depnds on $f_{y}, f_{y y}$ but is independent of $\varepsilon$.

In constructing the equidistributed interpolation error mesh, it is necessary to apply an extension of de Boor's theorem [9] to the present case of interpolating $f(x, y)$ with respect to $y$ for each $x$. This is a trivial extension since the piecewise linear approximation holds for any $x$. Thus the relation:

$$
\int_{z_{0}}^{z_{i}(x)}\left|f_{y y}(x, u)\right|^{1 / 2} d \xi=\frac{i}{N} \int_{z_{0}}^{z_{N}}\left|f_{y y}(x, \xi)\right|^{1 / 2} d \xi, \quad i=1,2, \cdots, N-1
$$

generates a family of curves with an equidistributed interpolation error. The curves are separated, and for any $x$, we have

$$
z_{0}<z_{1}(x)<z_{2}(x)<\cdots<z_{N-1}(x)<z_{N} .
$$


The choice of whether to use a uniformly spaced $z$-mesh or an equidistributed $z$-mesh must depend on $f(x, y)$. If $f$ is quadratic in $y$, the two criteria agree. In de Boor [11] it is shown that in using the equidistributed knots, the optimal bound $O\left(1 / N^{2}\right)$ is attained in some cases where the uniform knots are less than optimal. However, the uniform knots are easier to compute so we prefer them. This removes the remaining $N-1$ degrees of freedom discussed in $\S 2$.

In either case the mesh is determined by the solution and must be computed dynamically. An iterative scheme is introduced in the next section to construct the numerical solution and grid structure.

4. Adaptive mesh technique and solution of the algebraic system. We will now discuss the solution of the nonlinear system of equations $A(x, z) z=h(x, z)$ subject to $N-1$ additional relations. In the error analysis of the previous section, we have seen that it is desirable to have uniformly spaced $z_{i}$ points or to have equidistributed interpolation error. We will present an algorithm which solves this system along with the independent $N-1$ constraints that the $z_{i}$ be equally spaced. We will also discuss how to constrain the $z_{i}$ so that the interpolation error is equidistributed.

The first step is a fixed point iteration with a fixed $x$-mesh, say $x^{k}$. The solution $z^{k}$ of the "inner" iteration

$$
A\left(x^{k}, z^{k, m}\right) z^{k, m+1}=h\left(x^{k}, z^{k, m}\right)
$$

(where $m$ is an iteration counter) is an "acceptable" numerical solution of the boundary value problem. However, $z^{k}$ does not necessarily have the desirable error properties, namely, $|y-z|=O\left(N^{-2}\right)$. To achieve a uniformly spaced $z_{i}$, we update at the end of each "inner" iteration the value $x_{i}^{k+1}$. This entails the solution of a nonlinear equation:

$$
z^{k}\left(x_{i}^{k+1}\right)=z_{i}^{k}
$$

where

$$
z_{i}^{k}=i\left(\max z^{k}(x)-\min z^{k}(x)\right) / N .
$$

This procedure is called an "outer" iteration and amounts to an adaptive mesh technique. The $\left\{x_{i}^{k+1}\right\}$ is the new mesh. "Inner" and "outer" iterations are continued until convergence, i.e.,

$$
\left|x_{i}^{k+1}-x_{i}^{k}\right|<\varepsilon_{\text {Tol }}
$$

and (4.1) holds within $\varepsilon_{\text {Tol }}$, where $0<\varepsilon_{\text {Tol }} \ll 1$. Similarly, for a solution with equidistributed interpolation error we replace (4.1) by (3.3).

It remains to discuss how we obtain the initial guesses for $x, z$ based on $N$ intervals. We do this by solving a sequence of problems based on an increasing number of intervals: $N=1,2,4,8, \cdots, 2^{k}, \cdots$. The initial guess for $N=2^{k+1}$ intervals comes from the solution for $N=2^{k}$ intervals. By saving the previous solutions, we can perform Richardson extrapolation conveniently. Note that if $f$ is convex then the linear interpolations $I_{f}$ approach $f$ monotonically as $N$ increases. By Lemma 2, we then have that the corresponding solutions $z$ approach $y$ monotonically.

5. Construction of the elliptic splines. In the nonlinear system $\boldsymbol{A}(x, z) z=h(x, z)$, the matrix and right-hand side depend upon the derivatives of the spline basis functions $w_{i}$. In order to carry through the iteration scheme previously described, it is necessary to have a convenient and efficient way of computing these functions. The theory of asymptotics for linear ODE gives analytic representations $\left(w_{\varepsilon_{i}}^{\prime}\right)$ which, for $\varepsilon \rightarrow 0$ are asymptotic to the exact $w_{i}^{\prime}$. The representations are in terms of exponential functions 
of the $g_{1_{i}}$ and can be efficiently computed. Due to the asymptotic property, they become more accurate as $\varepsilon$ becomes smaller.

The following result is called the Liouville-Green or WKB approximation (Olver [29]). Let

$$
b_{i}^{ \pm}(x)=g_{1_{i}}^{-1 / 4}(x) \exp \left\{ \pm \frac{1}{\sqrt{\varepsilon}} \int g_{1_{i}}^{1 / 2}(x) d x\right\}
$$

Then there exist error functions $e^{+}, e$ such that $b^{+}\left(1+e^{+}\right), b^{-}\left(1+e^{-}\right)$are exact solutions of $E_{i} y=0$. On $x_{i-1}<x<x_{i}$ the error functions satisfy:

$$
\left|e_{i}^{ \pm}(x)\right| \leqq \exp \left\{\frac{\sqrt{\varepsilon}}{2} V_{ \pm}(F)\right\}-1, \quad F(x)=\int \frac{1}{g_{1_{i}}^{1 / 4}} \frac{d^{2}}{d x^{2}}\left(\frac{1}{g_{1_{i}}^{1 / 4}}\right) d x
$$

where $V_{ \pm}$is the variation of $F(x)$ between $x_{i-1}$ and $x_{i}$. Using these explicit $b_{i}^{ \pm}$we construct as before the approximations $u_{\varepsilon_{i}}, v_{\varepsilon_{i}}$ and eventually get $w_{\varepsilon_{i}}$ which are asymptotic approximations to the $\gamma$-elliptic splines $w_{i}$. These functions can be differentiated analytically and the results used to compute $A(x, z)$.

We introduce $S_{\varepsilon}\left(E,\left\{x_{i}\right\}\right)$, the space of $\gamma_{\varepsilon}$-elliptic splines, generated by the set $\left\{w_{\varepsilon_{i}}\right.$; $i=0,1, \cdots, N\}$. In general, $S_{\varepsilon}\left(E,\left\{x_{i}\right\}\right)$ approximates $S\left(E,\left\{x_{i}\right\}\right)$. In the important special case of an autonomous equation $\left(\varepsilon y^{\prime \prime}=f(y)\right), g_{1}$ is independent $x$ and the integration in (5.1) can be carried out exactly. Then the WKB approximation reduces to the familiar exponential basis (Berger et al., [7], deGroen and Hemker [23], deGroen [24], Hemker [25]) for a constant coefficient equation and is an exact solution of $E_{i} y=0$. For this case we therefore have $S_{\varepsilon}\left(E,\left\{x_{i}\right\}\right) \equiv S\left(E,\left\{x_{i}\right\}\right)$. If the integral in (5.1) cannot be evaluated exactly, a quadrature can be used. Since for many interesting problems $g_{1} \sim f_{y}(x, y)$ is a nice function of $x$ this quadrature can be done cheaply.

5.1. Construction of $y_{p}$. The asymptotic representation of the particular solution $y_{p_{i}}$ can be calculated by substituting the WKB representations of the local solutions into (2.9) and by performing integration by parts. This procedure yields a consistent expansion to all orders, but the amount of algebra to do these calculations can become prohibitive (Eckhaus [17]). However, the leading term is easily obtained:

$$
y_{p_{i}}=-G_{p_{i}}(x)+G_{p_{i}}\left(x_{i-1}\right) v_{\varepsilon_{i}}(x)+G_{p_{i}}\left(x_{i}\right) u_{\varepsilon_{i}}(x)+O(\varepsilon),
$$

where

$$
G_{p_{i}}(x)=g_{0_{i}} / g_{1_{i}}
$$

Alternatively we may use the results of Flaherty and O'Malley [20], Olver [29], O'Malley [33] in which the particular solution is decomposed into a solution $z_{i}$ of the reduced problem and boundary layer corrections $L_{i}, R_{i}$ :

$$
y_{p_{i}}(x)=L_{i}(x)+z_{i}(x)+R_{i}(x) .
$$

The solution $z_{i}$ is expanded as an asymptotic series in $\varepsilon$ and the coefficients are obtained recursively:

$$
\begin{aligned}
& z_{i}=\sum_{n=0} \varepsilon^{n} z_{i_{n}}(x), \\
& z_{i_{0}}(x)=-G_{p_{i}}(x), \\
& z_{i-1_{n}}^{\prime \prime}=g_{0_{i}}+g_{1_{i}} z_{i_{n}} .
\end{aligned}
$$


The boundary layer corrections $L_{i}, R_{i}$ are given by

$$
L_{i}(x)=-z_{i}\left(x_{i-1}\right) v_{\varepsilon_{i}}(x), \quad R_{i}(x)=-z_{i}\left(x_{i}\right) u_{\varepsilon_{i}}(x) .
$$

Note that $z_{i_{0}}(x)$ is the outer solution of (2.1). For the computations we have used only the leading term for $y_{p}$. This allows us to efficiently compute the right-hand side $h(x, y)$ of the algebraic system.

6. Numerical solutions. In this section, we present numerical solutions to a number of boundary value problems in the chemical and physical sciences.

Our first example is due to Carrier [14]. It arises in singular perturbation theory and geophysics:

$$
\varepsilon y^{\prime \prime}=\left[1-2 b\left(1-x^{2}\right) y-y^{2}\right] \quad \text { with } y^{\prime}(0)=0, \quad y(1)=0 .
$$

Note that when $b=0$, if $f_{y}(1,0)=0$ and, thus, $x=1$ and $y=0$ is a turning point of the problem. Since the WKB approximation breaks down near a turning point our method is, in principle, ineffective. However, because the chords which interpolate $f$ have nonzero slope, we can successfully compute the solution provided the number of mesh points is not large. For $b=0,(6.1)$ is autonomous and no such difficulties are encountered. Plotted in Fig. 6.1 is the graph of the solution for $\varepsilon=10^{-6}$ and $b=0$ with $N=4$. For this problem, the equidistributed interpolation error mesh is identical to a uniform $y$-mesh since $f(x, y)$ is a quadratic function of $y$. In Table 6.1, the value $x(y=-.5)$ is given as a function of $N$, the number of intervals, using $N=64$ solution

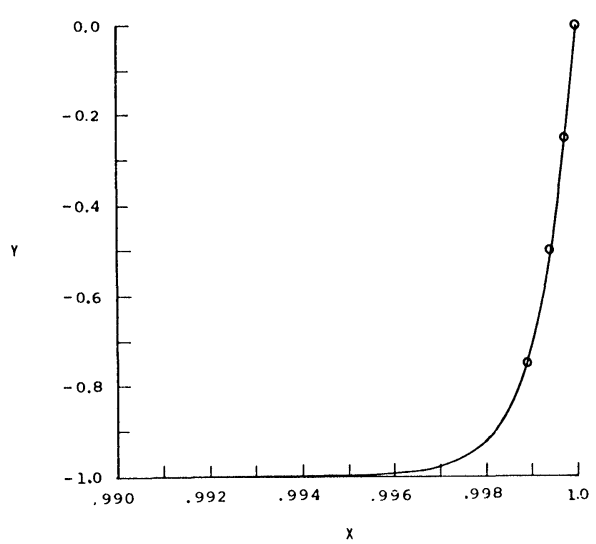

FiG. 6.1. Solution of Carrier's problem with $\varepsilon=10^{-6}, b=0$ and $N=4$.

TABLE 6.1

Carrier's problem with $\varepsilon=10^{-6}$

\begin{tabular}{rcccc}
\hline$N$ & $x_{N}(y=0.5)$ & $\left|x_{N}-x_{64}\right| \times 10^{5}$ & $e_{N} \times 10^{5} \times N^{2}$ & $\begin{array}{c}\text { Extrapolated } \\
\text { value }\end{array}$ \\
\hline 2 & .99941385 & 2.29 & 9.16 & \\
4 & .99943121 & .554 & 8.86 & .999437 \\
8 & .99943538 & .137 & 8.77 & .99943677 \\
16 & .99943642 & .033 & 8.43 & .99943677 \\
32 & .99943668 & .007 & 7.17 & \\
64 & .99943675 & .0 & & \\
\hline
\end{tabular}


as a representation of the exact solution. The error in the location of $x(y=-.5)$ is tabulated in the second column. Column 3 shows that the error in locating $y=-.5$ is, indeed, $O\left(1 / N^{2}\right)$. Finally, Richardson's extrapolation is applied to the results and is tabulated in column 5. For $b=1$, we plot the solution in Fig. 6.2 for $\varepsilon=10^{-6}$ and $N=8$. Note that our method computes successfully the transition between the outer solution and the boundary layer with only a few grid points (see Fig. 6.3). This attests to the importance of using a local basis that captures the essential behavior of the solution.

The second example is the Troesch problem modeling the confinement of a plasma column by radiation pressure [44]:

$$
y^{\prime \prime}=\varphi \sinh \varphi y, \quad \varphi>0, \quad y(0)=0, \quad y(1)=1 .
$$

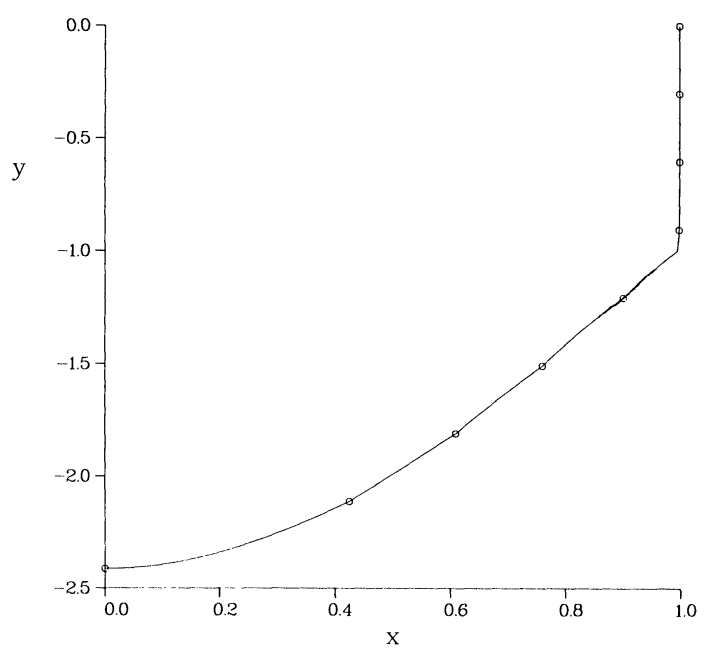

FIG. 6.2. Solution of Carrier's problem with $\varepsilon=10^{-6}, b=1$ and $N=8$.

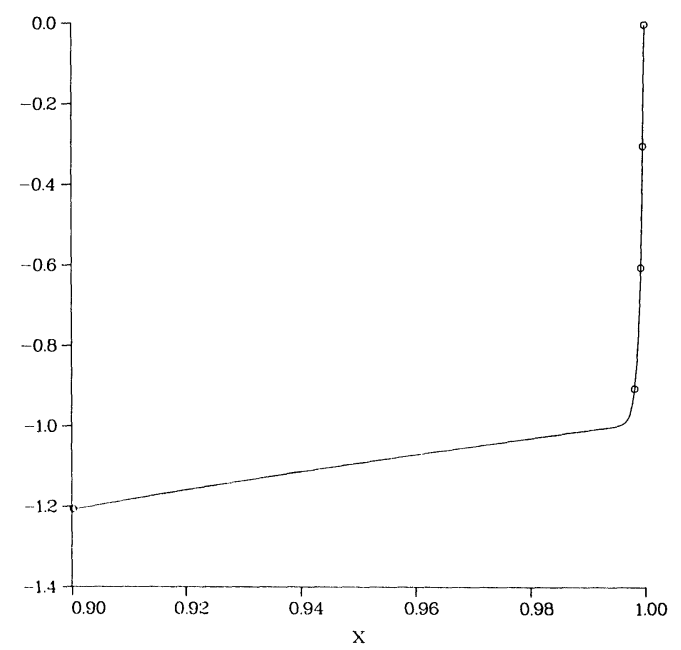

FIG. 6.3. An enlarged graph of Fig. 6.2. for $.90 \leqq x \leqq 1.00$. 
This is a particularly difficult problem because of the strongly nonlinear right-hand side. The problem of computing $y^{\prime}(0)$ and $y^{\prime}(1)$ has been used as a benchmark for stiff boundary value problem algorithms (Roberts and Shipman [39], Troesch [44]). The derivatives $y^{\prime}(0)$ and $y^{\prime}(1)$ have the following asymptotic expansion (Anglesio and Troesch [2], Chin [15]):

$$
y^{\prime}(0)=y_{0}^{\prime}(0)\left\{1+\frac{y_{0}^{\prime}(0)^{2}}{4}\left[\varphi-1+\frac{\cosh (\varphi / 2)}{\sinh ^{2}(\varphi / 2)}\right]\right\}+O\left(\varphi e^{-3 \varphi}\right)
$$

where

$$
y_{0}^{\prime}(0)=8 e^{-\varphi} \tanh (\varphi / 4)
$$

and

$$
y^{\prime}(1)=2 \sinh (\varphi / 2)\left[1-\left\{y^{\prime}(0) / 2 \sinh (\varphi / 2)\right\}^{2}\right]^{1 / 2} .
$$

We plot in Fig. 6.4 the relative error in the derivative $y^{\prime}(0)$ between the numerical and the asymptotic solution (6.2) multiplied by $N^{2}$ as a function of $N$ for $\varphi=1,5$, 10,20 . The $N^{2}$ normalization is suggested by the error analysis of $\S 3$. The computations are done with an uniformly spaced $y$-mesh. Also plotted in Fig. 6.4 is a calculation for $\varphi=5$ using a equidistributed interpolation error mesh. Figure 6.5 is a similar plot for the derivative $y^{\prime}(1)$. It is seen from Figs. 6.4-6.5 that $y^{\prime}(1)$ is calculated more accurately than $y^{\prime}(0)$ as $\varphi$ increases. This is because the mesh points collect in the boundary layer with increasing $\varphi$. Moreover, the equidistributed interpolation error

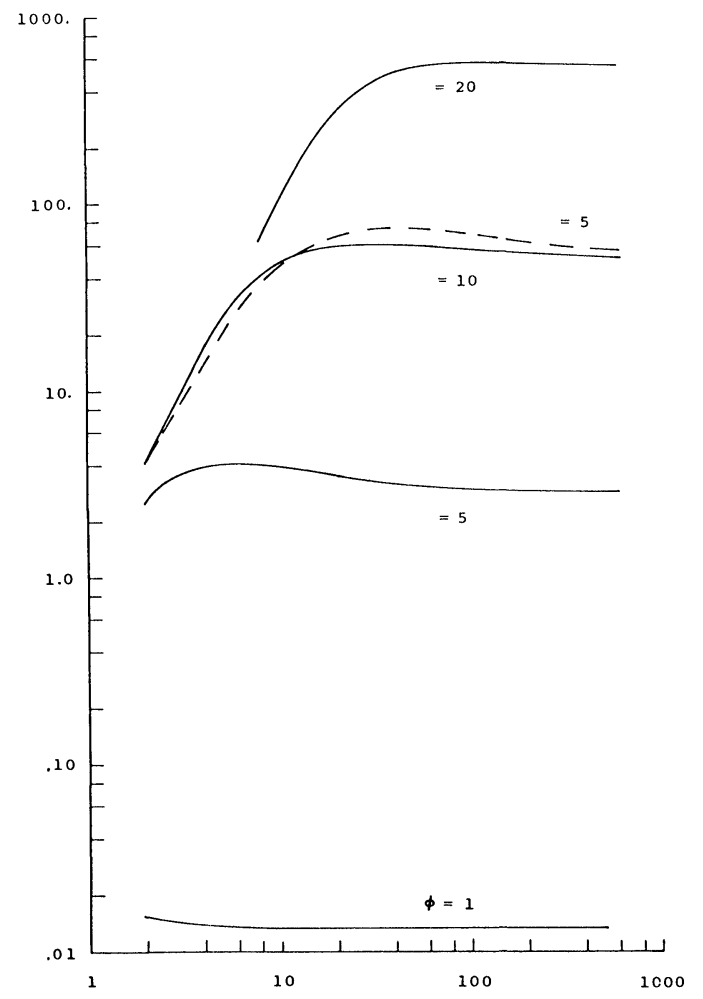

FIG. 6.4. Solution of Troesch problem: Relative error of $y^{\prime}(0),\left[z^{\prime}(0)-y^{\prime}(0)\right] N^{2} / y^{\prime}(0)$ as a function of the number of intervals, $N$. Equidistribution of interpolation error is used (--). 


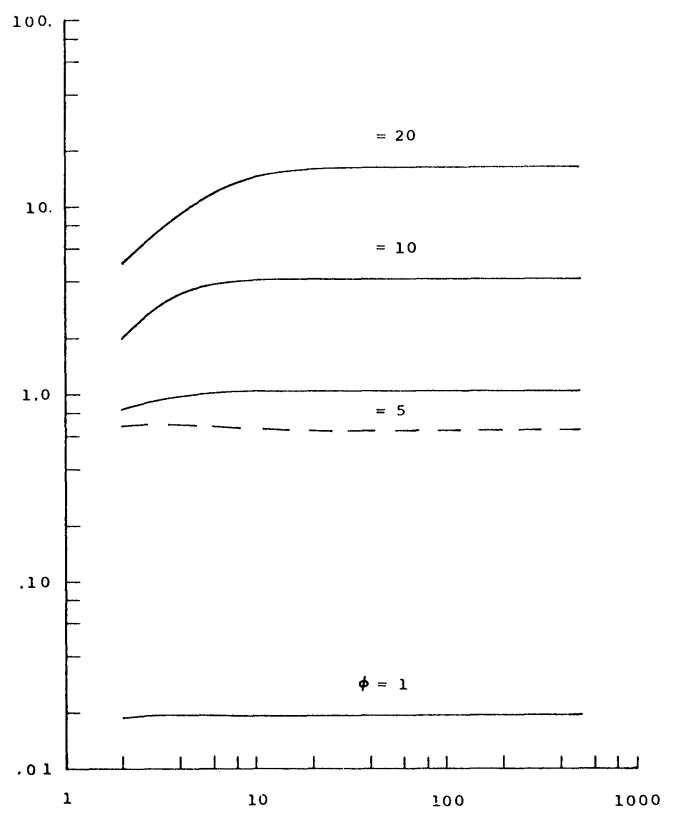

FIG. 6.5. Solution of Troesch problem: Relative error of $y^{\prime}(1),\left[z^{\prime}(1)-y^{\prime}(1)\right] N^{2} / y^{\prime}(1)$ as a function of the number of intervals, $N$. Equidistribution of interpolation error is used (--).

mesh tends to locate more points in the boundary layer than the uniformly spaced $y$-mesh. As a consequence, the equidistributed interpolation error mesh yields a more accurate value of $y^{\prime}(1)$ while the uniformly spaced $y$-mesh gives a better approximation to $y^{\prime}(0)$. Overall if both derivatives $y^{\prime}(0)$ and $y^{\prime}(1)$ are desired, the uniformly spaced $y$-mesh tends to perform better.

Our final example appears in the theory of diffusion and reaction in permeable catalysts (Aris [3]):

$$
\begin{aligned}
& \frac{d^{2} c}{d x^{2}}=\varphi^{2} \exp \left\{\gamma\left(1-\frac{1}{T}\right)\right\} f(c), \\
& \frac{d^{2} T}{d x^{2}}=-\beta \varphi^{2} \exp \left\{\gamma\left(1-\frac{1}{T}\right)\right\} f(c) .
\end{aligned}
$$

with boundary conditions

$$
c^{\prime}(0)=T^{\prime}(0)=0, \quad c(1)=1-\frac{1}{S h} c^{\prime}(1),
$$

and

$$
T(1)=1-\frac{1}{N u} T^{\prime}(1)
$$

Equations (6.4) may be combined with boundary conditions (6.5) to yield

$$
T(x)=1+\beta \frac{S h}{N u}+\beta\left[1-\frac{S h}{N u}\right] c(1)-\beta c(x) .
$$

Substituting (6.6) into (6.4), we obtain an equation for $c$. However, the boundary value $c(1)$ appears in the differential equation and, thus, complicates the solution 
procedure. We solve $(6.4 \mathrm{a}),(6.6)$ and boundary conditions $(6.5)$ using the parameters given by Carey and Finlayson [13]:

$$
\beta=0.2, \quad S h / N u=50, \quad S h=250, \quad \varphi=14.44, \quad \gamma=20 \quad \text { and } f(c)=c .
$$

The most important feature of the solution is the accurate computation of $d T / d x(1)$ (Carey and Finlayson [13]). In view of the results on the Troesch problem and of the added expense in applying the equidistributed interpolation error mesh, a uniformly spaced $y$-mesh is selected for this set of computations.

The solution for $N=4$ is plotted in Fig. 6.6. The value $d T / d x(1)$ is tabulated in Table 6.2 as a function of $N$. It is clearly seen from Table 6.2 that four place accuracy in $d T / d x(1)$ is obtained with just two intervals.

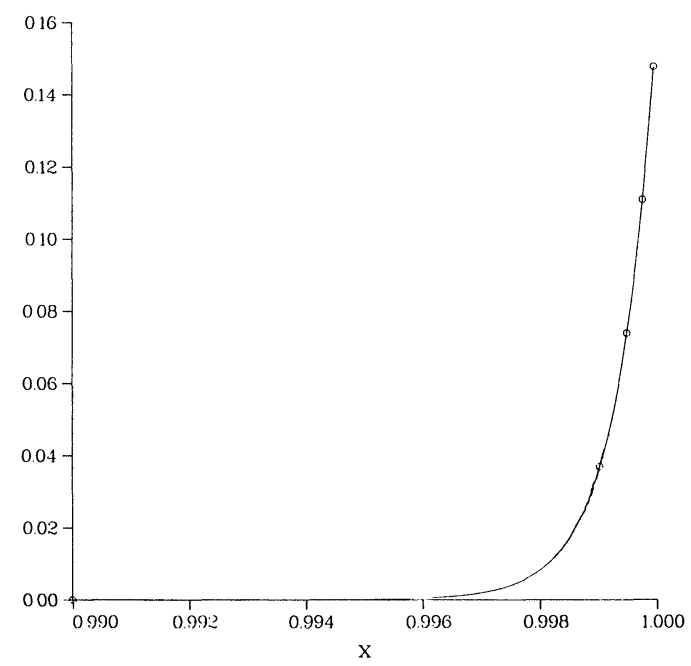

FIG. 6.6. Solution of a diffusion-reaction equation with $N=4$.

TABLE 6.2

Solution of a diffusion-reaction equation

\begin{tabular}{rc}
\hline$N$ & $d T / d x(1)$ \\
\hline 1 & -4.2586013 \\
2 & -4.2607540 \\
4 & -4.2612872 \\
8 & -4.2613201 \\
16 & -4.2614534 \\
32 & -4.2614617 \\
64 & -4.2614637 \\
\hline
\end{tabular}

7. Conclusions and generalizations. It can be seen from the numerical examples just how important is the use of a basis that captures the essential behavior of the solution. The basis functions must reflect the rapid growth or decay of the solution. This property is inherent in asymptotic methods and in solving boundary value problems by approximating the coefficients. 
Since the class of problems we have considered is rather restricted, we close with some suggestions about extensions of the method to a wider class. For problems with $f$ depending on $y$ and $y^{\prime}$, a multivariate interpolation with respect to $y$ and $y^{\prime}$ on a triangular domain may be used to reduce the nonlinear problem to a collection of disjointed linear two-point boundary value problems. The same end can be achieved by combining a straightforward application of quasilinearization and approximating the coefficients of the linear differential equation. The subinterval problems are then solved by asymptotic methods. The local solutions are "patched" together to form the global solution. Here, the WKB method is invalid near turning points. This calls for a uniform asymptotic method in the neighborhood of a turning point.

For a system of differential equations, quasilinearization followed by approximating the coefficients of the linear DE is more suitable. The resulting linear boundary value problem in each subinterval, however, is by no means trivial to solve.

Acknowledgments. We thank Gerald Hedstrom for many stimulating discussions. We also thank the U.S. Department of Energy Office of Basic Energy Sciences, Applied Mathematics and Statistics Division. We thank J. E. Flaherty and G. J. Majda for suggesting improvements in the presentation of the manuscript.

\section{REFERENCES}

[1] L. R. Abrahamsson, H. B. Keller AND H. O. Kreiss, Difference approximations for singular perturbations of systems of ordinary differential equations, Numer. Math., 22 (1974), pp. 367-391.

[2] J. D. ANGLESIO AND B. A. TROESCH, High precision results for a two-point boundary value problem, J. Comp. Appl. Math., 6 (1980), pp. 99-103.

[3] R. ARIS, The Mathematical Theory of Diffusion and Reaction in Permeable Catalysts, Vol. I, Clarendon Press, Oxford, 1975.

[4] U. Ascher, J. Christiansen And R. D. Russell, A collocation solver for mixed order systems of boundary value problems, Math. Comp., 33 (1979), pp. 659-679.

[5] J. P. Aubin, Approximation of Elliptic Boundary-Value Problems, Wiley-Interscience, New York, 1972, Chapt. 6.

[6] A. E. Berger, J. M. Solomon, M. Ciment, S. H. Leventhal and B. C. Weinberg, Generalized OCI schemes for boundary layer problems, Math. Comp., 35 (1980), pp. 659-731.

[7] A. E. Berger, J. M. Solomon AND M. Ciment, An analysis of uniformly accurate difference method for a singular perturbation problem, Math. Comp., 37 (1981), pp. 79-94.

[8] S. R. Bernfeld And V. LAkshmikantham, An Introduction to Nonlinear Boundary Value Problems, Academic Press, New York, 1974.

[9] C. DE BOOR, Good approximation by splines with variable knots, in Spline Functions and Approximation Theory. A. Muir and A. Sharma, eds., Birkhauser Verlag, Basel, 1973, pp. 52-72.

[10] C. DE BOOR AND B. SWARTZ, Collocation at Gaussian points, SIAM J. Numer. Anal., 10 (1973), pp. 583-606.

[11] C. DE BOOR, A Practical Guide to Splines, Springer-Verlag, New York, 1978.

[12] N. I. BRIS, On boundary problems for the equation $\varepsilon y^{\prime \prime}=f\left(x, y, y^{\prime}\right)$ for small $\varepsilon$, Dokl. Akad. Nauk. SSSR, 95 (1954), pp. 429-432. (In Russian.)

[13] G. F. CARey AND B. A. Finlayson, Orthogonal collocation on finite elements, Chem. Engr. Sci., 30 (1975), pp. 587-596.

[14] G. F. CARRIER, Singular perturbations and geophysics, SIAM Rev., 12 (1970), pp. 175-193.

[15] R. C. Y. CHIN, On the matched asymptotic solution of the Troesch problem, J. Comp. Appl. Math., 7 (1981), 181-185.

[16] F. W. Dorr, S. V. Parter ANd L. F. Shampine, Applications of the maximum principle to singular perturbation problems, SIAM Rev., 15 (1973), pp. 43-88.

[17] W. ECKHAus, Asymptotic Analysis of Singular Perturbations, North-Holland, Amsterdam, 1979.

[18] P. C. FIFE, Semilinear elliptic boundary value problems with small parameters, Arch. Rational Mech. Anal., 52 (1973), pp. 205-232, MR51 \#10863.

[19] J. E. FLAHERTY AND W. MATHON, Collocation with polynomial and tension splines for singularlyperturbed boundary value problems, this Journal, 1 (1980), pp. 260-289. 
[20] J. E. Flaherty AND R. E. O'MALley, JR, The numerical solution of boundary value problems for stiff differential equations, Math. Comp., 31 (1977), pp. 66-93.

[21] B. Friedman, Principles and Techniques of Applied Mathematics, John Wiley, New York, 1956.

[22] D. Gidaspow AND B. S. BAKER, A model for discharge of storage batteries, J. Electrochem. Soc., 120 (1973), pp. 1005-1010.

[23] P. P. N. DE GROEN AND P. W. HEMKER, Error bounds for exponentially fitted Galerkin methods applied to stiff two-point boundary value problems, in Numerical Analysis of Singular Perturbation Problems, P. W. Hemker and J. J. H. Miller, eds., Academic Press, London, 1979.

[24] P. P. N. DE GROEN, A finite element method with a large mesh-width for a stiff two-point boundary value problem, J. Comp. Appl. Math., 7 (1981), pp. 3-15.

[25] P. W. HeMKer, A numerical study of stiff two-point boundary problems, Ph.D. dissertation, Mathematisch Centrum, Amsterdam, 1977.

[26] H. B. Keller, Numerical Methods for Two-Point Boundary Value Problems, Ginn-Blaisdell, Waltham, MA, 1968.

[27] R. B. Kellog AND A. Tsan, Analysis of some difference approximations for a singular perturbation problem without turning points, Math. Comp., 32 (1978), pp. 1025-1039.

[28] V. S. Markin, A. A. Chernenko, Yu. A. Chizmadzhev and Yu. G. Chirkov, Aspects of the theory of gas porous electrodes, in Fuel Cells, Their Electrochemical Kinetics, V. S. Bagotskii and Yu. B. Vasil'ev, eds., Consultants Bureau, New York, 1966, pp. 21-33.

[29] F. W. J. Olver, Asymptotics and Special Functions, Academic Press, New York, 1974.

[30] —- Asymptotic approximations and error bounds, SIAM Rev., 22 (1980), pp. 188-203.

[31] R. E. O'MALley, JR, On a boundary value problem for a nonlinear differential equation with a small parameter, SIAM J. Appl. Math., 17 (1969), pp. 569-581.

[32] - Introduction to Singular Perturbations, Academic Press, New York, 1974.

[33] - Boundary layer methods for ordinary differential equations with small coefficient multiplying the highest derivatives, Proc. Sympos. on Constructive and Computational Methods for Differential and Integral Equations, Lecture Notes in Mathematics 430, Springer-Verlag, New York, 1974, pp. 363-389.

[34] S. OSHER, Numerical solution of singular perturbation problems and hyperbolic systems of conservation law, in Analytical and Numerical Approaches to Asymptotic Problems in Analysis, S. Axelsson, L. S. Frank, and A. Van Der Sluis, eds., North-Holland, Amsterdam, 1981, pp. 179-204.

[35] M. H. Protter And H. F. Weinberger, Maximum Principles in Differential Equations, PrenticeHall, Englewood Cliffs, NJ, 1967.

[36] S. A. PRuESS, Solving linear boundary value problems by approximating the coefficients, Math. Comp., 27 (1973), pp. 551-561.

[37] G. W. REDDIEN, Projection methods for two-point boundary value problems, SIAM Rev., 22 (1980), pp. 156-171.

[38] M. E. Rose, Finite difference schemes for differential equations, Math. Comp., 18 (1964), pp. 179-195, MR32 \# 605.

[39] S. M. Roberts And J. S. Shipman, On the closed form solution of Troesch's problem, J. Comp. Phys., 21 (1976), pp. 291-304.

[40] R. D. Russell ANd L. F. Shampine, A collocation method for boundary value problems, Numer. Math., 19 (1972), pp. 1-28.

[41] M. H. SCHultz, Elliptic spline functions and the Rayleigh-Ritz-Galerkin method, Math. Comp., 24 (1970), pp. 65-80.

[42] L. F. SHAMPINE, Boundary value problems for ordinary differential equations. II: patch bases and monotone methods, SIAM J. Numer. Anal., 6 (1969), pp. 414-431.

[43] I. STAKgold, Boundary Value Problms of Mathematical Physics Vol. I, Macmillan, New York, 1967.

[44] B. A. Troesch, A simple approach to a two-point boundary value problem, J. Comp. Phys., 21 (1976), pp. 279-290.

[45] J. YARMISH, Newton's method techniques for singular perturbations, SIAM J. Math. Anal., 6 (1975), pp. 661-680. 